\title{
Comparison of interleukin expression (interleukin-2 and interleukin-9) in chronic otitis media with and without cholesteatoma
}

\author{
Jose A. Marino-Silva* and Israel A. Espinosa-Rey \\ Servicio de Otorrinolaringología, Hospital General de México, Mexico City, Mexico
}

\begin{abstract}
Introduction: Chronic suppurative otitis media (CSOM) is a persistent inflammation of the middle ear, with cholesteatoma as one of its many complications. Cholesteatoma is a progressive, non-neoplastic formation of keratinized squamous epithelium that causes bone destruction in the middle ear. There are multiple theories that may explain its physiopathogenesis; however, none of them have been proved yet. Inflammatory mediators, such as interleukins (ILs), have a role in the genesis of the cholesteatoma and it is necessary to study their role in detail. Objective: The objective of this study was to compare the IL (IL-2 and IL-9) expression in patients with CSOM with and without cholesteatoma. Methodology: A cross-sectional study, comparing two groups of patients with CSOM, five of them without cholesteatoma and eleven with cholesteatoma. Samples of problem tissue (inflammatory tissue and cholesteatoma) and control tissue (retroauricular tissue for both groups) were studied. Histopathological and immunohistochemistry analyses were conducted, in addition to imaging and morphometric analysis was performed. The statistical comparison was performed by $t$-test for independent groups. Conclusions: There are statistical differences on the IL-2 and IL-9 expression in the cholesteatoma tissue compared with the inflammatory tissue.
\end{abstract}

Key words: Cholesteatoma. Chronic suppurative otitis media. Interleukins. Interleukin-2. Interleukin-9.

\section{Introduction}

Otitis media (OM) are the inflammation of the middle ear and mastoid cells. This inflammation can be due to various factors such as foreign bodies, chemical substances, or infection. If the inflammation lasts longer than 6 weeks, accompanied by ear discharges (otorrhea), without fever or pain, then OM is considered chronic suppurative OM (CSOM). CSOM is characterized by an insidious, progressive onset and in case of potential complications; it can lead to sequelae'.

Complications secondary to CSOM are more frequent in adult patients. These are divided into intracranial and extracranial complications. As for intracranial complications, the most common is meningitis. It can present itself as hematogenous dissemination from the subarachnoid space, by contiguity of the middle ear through Hyrtl's fissure or by bone erosion and, finally, by direct extension. Other intracranial complications include brain abscess, thrombosis of the lateral sinus, or sigmoid sinus 1 .

Extracranial complications are subdivided into extratemporal and intratemporal complications. Extratemporal complications include subperiosteal abscess, Bezold's abscess, and deep neck abscess. Intratemporal complications include cholesteatoma, labyrinthine fistula, cochlear fistula, mastoiditis, petrous apicitis, and

\section{Correspondence:}

*Jose A. Marino-Silva

E-mail: drmarino88@gmail.com
Date of reception: 27-07-2017

Date of acceptance: 31-08-2017
Available online: 13-09-2019 Rev Med Hosp Gen Mex. 2019;82(3):119-124 www.hospitalgeneral.mx under the CC BY-NC-ND license (http://creativecommons.org/licenses/by-nc-nd/4.0/). 
facial paralysis. Labyrinthine fistula is primarily associated with cholesteatoma, in affecting the horizontal semicircular canal $90 \%$ of the time ${ }^{2}$.

Cholesteatoma is the main intratemporal complication $^{2}$. Cholesteatoma is a non-neoplastic lesion with destructive properties. It is characterized by the migration of keratinized squamous epithelium with ciliated pseudostratified columnar epithelium lining, in a fibrous stroma within the middle ear and the mastoid cavity. The existence of molecular dysregulation involving inflammatory mediators and growth factors has been detected in these cases $^{3}$. The incidence of cholesteatoma in adults varies according to the population, ranging from 4.2 to $9.2 / 100,000$ adults and is 1.4 times more common in men than in women 4 .

Cholesteatoma can be primary when it occurs in children with intact tympanic membrane. Primary acquired cholesteatoma is the presence of a secondary diverticulum in the pars flaccida and secondary acquired cholesteatoma occurs when it is associated with perforation of the tympanic membrane. In this article, acquired cholesteatoma is studied.

There are multiple theories that attempt to explain the physiopathogenesis of acquired cholesteatoma, theories related to metaplasia, migration, basal hyperplasia, iatrogenesis, and retraction pocket, to name a few ${ }^{5}$. However, none of these theories has been proven until today or is deemed to have a multifactorial origin ${ }^{6}$. It has been proven that the degree of inflammation responds to the aggression to which the middle ear is subjected, as in the case of a repetitive bacterial superinfection?.

The immune system responds to lesions or irritations that occur in the body through an inflammation cascade. As part of this inflammation, cascade, epithelial cells, endothelial cells, and, mainly, inflammatory cells (mononuclear cells, macrophages, lymphocytes, and mast cells) ${ }^{3}$ produce inflammatory mediators. Molecularly, inflammatory mediators can be proteins, glycoproteins, peptides, cytokines, and others. Although the primary objective of inflammatory mediators is to protect the host, it can also cause damage, such as cholesteatoma in OM.

The etiopathogenesis of cholesteatoma is not yet clear; however, the participation of different inflammatory mediators as a central mechanism for its appearance has been studied ${ }^{3}$. Cytokines are among the main inflammatory mediators. Cytokines are low-molecular-weight glycoproteins that allow intercellular communication, through which immune responses are stimulated or suppressed and participate in the healing of wounds and tissue remodeling.
The cytokines involved in acquired cholesteatoma, which are mentioned in literature, are interleukin (IL)-1, IL-5, IL-6, IL-8, interferon-gamma (IFN-g) and tumor necrosis factor-alpha, tissue growth factor alpha and beta, and nitric oxide ${ }^{3,8,9}$. Another element present in the cholesteatoma is cytokeratins (CKs), due to their hyperproliferative properties. CKs 1, 2, 5, 10, and 14 have been found in cholesteatoma ${ }^{8}$.

\section{IL-2}

IL-2 is a chain of 133 polypeptides produced by T-lymphocytes; its main function is to initiate the proliferation and differentiation of T-lymphocytes. IL-2 is necessary to generate $\mathrm{CD} 8^{+} \mathrm{T}$-cells and increases the function of natural killer (NK) cells ${ }^{3,10}$.

\section{IL-9}

IL-9 is produced by T-lymphocytes, stimulates the release of mast cell mediators, and promotes the expression of high-affinity IgE receptors. It also promotes mucosal hypersecretion and maturation of eosinophils in conjunction with IL-53.

At present, the use of imaging methods has allowed greater certainty in the diagnosis of cholesteatoma, especially computed axial tomography and magnetic resonance imaging, in $\mathrm{T} 1$ diffusion-weighted sequences ${ }^{11,12}$.

Few studies suggest the medical treatment of cholesteatoma in the reviewed literature. The medical treatment of choice is generally surgery.

\section{Objectives}

The objectives of this study were to compare the expression of IL-2 and IL-9 in patients with CSOM with or without cholesteatoma.

\section{Specific objectives}

The specific objectives of this study were as follows:

- To determine the expression of IL in cholesteatoma, using immunohistochemistry with morphometric measurement technique by image analysis

- To evaluate morphometry by image analysis for the measurement of IL in cholesteatoma.

\section{Justification}

CSOM and its complications pose a great challenge in ENT consultations, being one of the most frequent 
reasons for medical appointments. The pathogenesis of cholesteatoma has been studied for more than 20 years and is still not fully understood, although it has been observed that a large number of inflammatory markers are involved, including IL. We consider that, when $\mathrm{IL}$ are detected in the inflammatory tissue at an early stage, it can be treated in a timely manner by specific inhibitors to prevent complications.

\section{Methodology}

This is a cross-sectional, descriptive study conducted in surgical patients suffering from CSOM with or without cholesteatoma at the Otorhinolaryngology and Head and Neck Surgery service of the General Hospital of Mexico (Hospital General de México). Inclusion criteria were as follows: patients aged 18 years or older, of both sexes, having a computed tomography scan of the ear in axial and coronal planes in a simple phase with data compatible with the diagnosis, who agreed to participate in the study and signed the informed consent form. Exclusion criteria included patients with neoplastic diseases, comorbidities with systemic inflammatory diseases, and those who had medical contraindications for surgical treatment. Elimination criteria were for tissue sampling that was not performed systematically and appropriately, and for those whose biopsy results were not compatible with CSOM diagnosis.

Regarding the 16 patients studied, five patients had no cholesteatoma (Group A) and 11 of them presented cholesteatoma (Group B). In both groups, the expression of IL of two comparative tissue biopsies was determined, the first (problem tissue) of the mastoid antrum for Group A and cholesteatoma for Group B. Skin biopsy control of the retroauricular region was performed in all cases (control tissue).

The samples were sent to histopathological tissue analysis in $10 \%$ formalin buffer. For the analysis, paraffin was included and $3 \mu \mathrm{m}$ cuts were made. For the macroscopic study, hematoxylin-eosin was stained and Masson's trichrome stain was used to determine fibrosis and changes related to chronicity. They were incubated with IL-2 and IL-9 antibodies and analyzed with conventional $A B C$ immunohistochemistry technique.

Diaminobenzidine chromogen was used for ochre brown staining as positive and this was compared with a negative control incubated in normal sheep serum. In all samples, anti-CK 13 antibody was incubated to corroborate the presence of cholesteatoma.

For comparison of CSOM with or without cholesteatoma, cholesteatoma was evidenced histologically in

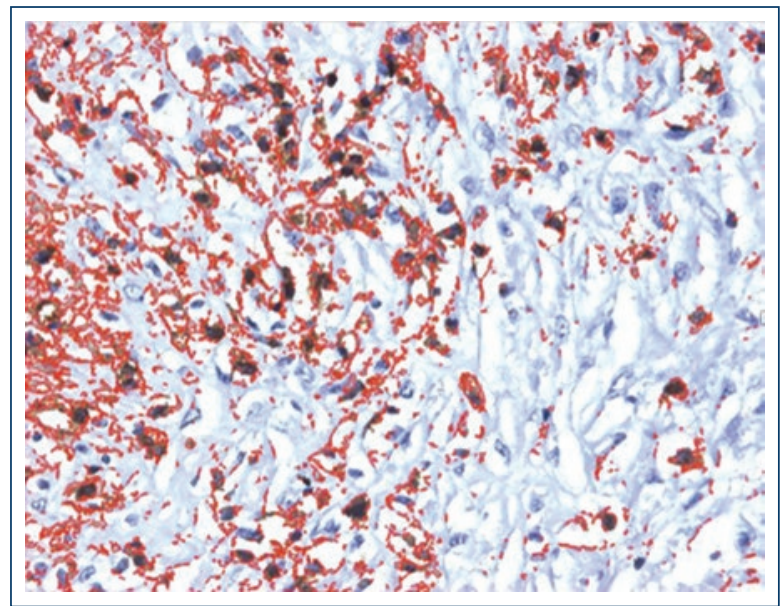

Figure 1. Group A micrograph analyzed with Image-Pro Plus 5.1 showing poor expression of interleukin-2.

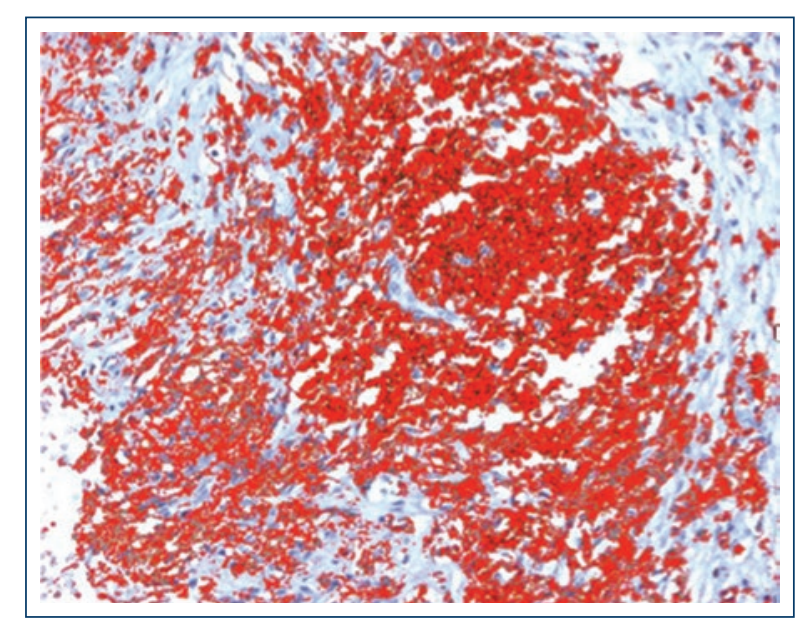

Figure 2. Group B micrograph analyzed with Image-Pro Plus 5.1 with high expression of interleukin-2.

the presence of continuous stratified squamous epithelium or fragments thereof that included keratin layers with a cystic center. Moreover, positive immunohistochemistry for CK-13 was confirmed. The diagnosis of mastoiditis was achieved histologically with the presence of acute and/or chronic inflammatory tissue, granulation tissue and/or necrosis without stratified squamous epithelium, and typical of cholesteatoma; diagnosis was confirmed with the immunohistochemical absence of CK-13.

The microscopic sections were digitized and standardized and later analyzed using an image processor, with Image-Pro Plus 5.1 software. The number of pixels of the positive samples was converted into numerical values. The differences in the analysis of the tissue image generated by software are shown in figures 1 and 2 . 


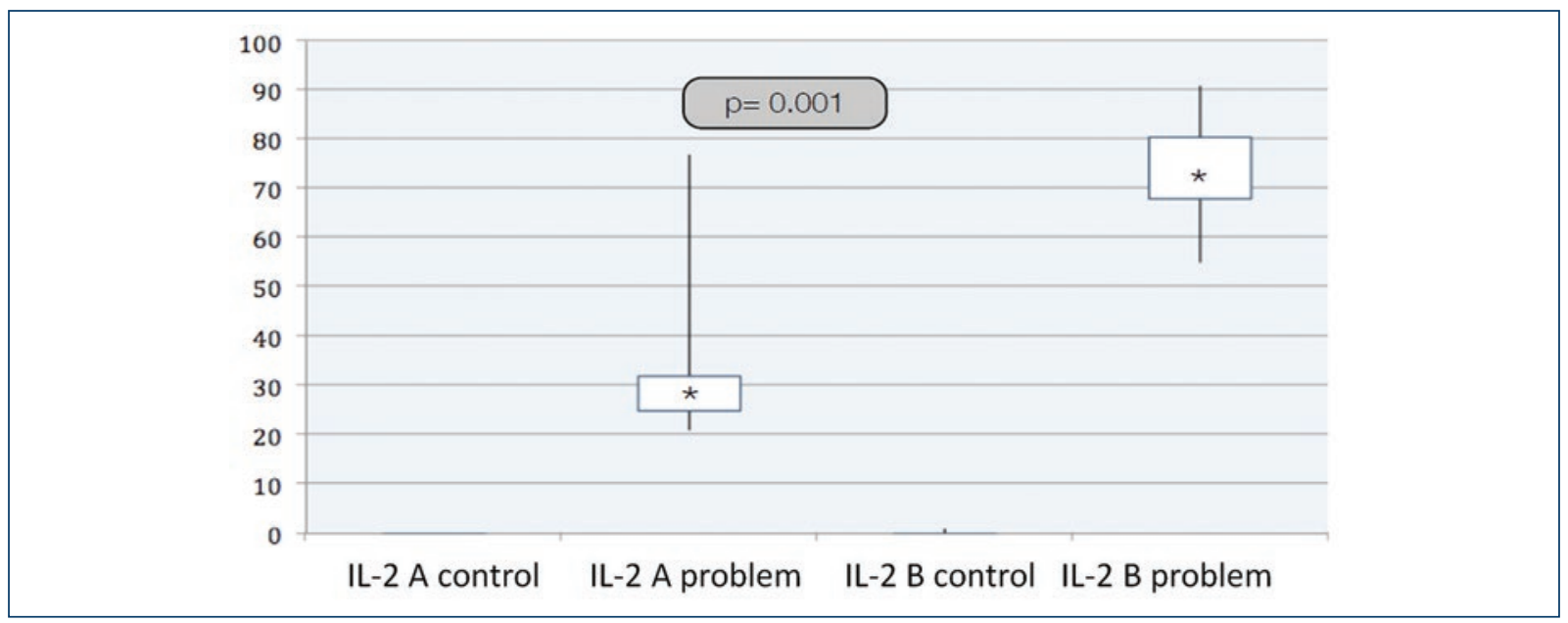

Figure 3. Interleukin-2 comparison, both groups.

Table 1. Measurement of cytokine expression in individuals

\begin{tabular}{|c|c|c|c|c|c|c|}
\hline \multirow{2}{*}{} & \multicolumn{2}{|c|}{ CK-13 } & \multicolumn{2}{c|}{ IL-2 } & \multicolumn{2}{c|}{ IL-9 } \\
\cline { 2 - 7 } & C* & P** & C* & P** & C* & P** \\
\hline Group A & & & & & & \\
\hline 1 & 1.08 & 0 & 0.00 & 0.52 & 0.17 & 0.23 \\
\hline 2 & 1.22 & 0.14 & 0.00 & 0.48 & 0.17 & 0.27 \\
3 & 1.43 & 0.1 & 0.00 & 0.60 & 0.17 & 0.35 \\
\hline 4 & 0.88 & 0.1 & 0.00 & 1.07 & 0.14 & 0.14 \\
5 & 1.06 & 0 & 0.00 & 0.56 & 0.10 & 0.34 \\
\hline Group B & & & & & & \\
\hline 1 & 1.23 & 0.72 & 0.00 & 0.87 & 0.23 & 0.64 \\
\hline 2 & 1.47 & 0.85 & 0.00 & 1.07 & 0.20 & 0.75 \\
3 & 1.2 & 0.79 & 0.00 & 1.04 & 0.17 & 0.62 \\
\hline 4 & 0.94 & 0.4 & 0.00 & 1.02 & 0.20 & 0.73 \\
\hline 5 & 1.43 & 0.73 & 0.00 & 0.97 & 0.20 & 0.83 \\
\hline 6 & 1.17 & 0.62 & 0.00 & 1.27 & 0.00 & 0.85 \\
\hline 7 & 1.37 & 0.61 & 0.00 & 0.97 & 0.00 & 0.59 \\
\hline 8 & 1.32 & 0.54 & 0.00 & 0.84 & 0.10 & 0.54 \\
\hline 9 & 1.47 & 0.59 & 0.00 & 1.16 & 0.10 & 0.59 \\
\hline 10 & 1.23 & 0.71 & 0.00 & 1.15 & 0.17 & 0.51 \\
\hline 11 & 1.07 & 0.48 & 0.10 & 1.08 & 0.10 & 0.45 \\
\hline
\end{tabular}

$C^{*}$ : Control tissue. In both groups, it corresponds to retroauricular skin. $\mathrm{P}^{* *}$ : Problem tissue: in Group $A$, it corresponds to inflammatory tissue of the middle ear. In Group B, it corresponds to cholesteatoma, IL-2: interleukin-2, IL-9: interleukin-9.

The statistical analysis was conducted on a comparison test of independent groups with t-test.

\section{Results}

Paired samples from 16 patients were analyzed; nine women and seven men, with a mean age of 42.5 years ( \pm 12.5 years), in a range of $18-58$ years (median: 41.4 years).
Table 2. Expression of cytokines in both groups

\begin{tabular}{|l|c|c|c|c|c|}
\hline & \multicolumn{2}{|c|}{ Group A } & \multicolumn{2}{c|}{ Group B } & \multirow{2}{*}{ P } \\
\cline { 2 - 5 } & C* & P** & C* & P** & \\
\hline CK-13 & 79.8 & 0.8 & 88.9 & 35.9 & $<0.001$ \\
\hline IL-2 & 0 & 36.6 & 0.09 & 73.5 & 0.001 \\
\hline IL-9 & 2.4 & 7.4 & 2.3 & 36.4 & $<0.001$ \\
\hline
\end{tabular}

$C^{*}$ : Control tissue. In both groups, it corresponds to retroauricular skin. $P^{* *}$ : Problem tissue: in Group A, it corresponds to inflammatory tissue of the middle ear. In Group B, it corresponds to cholesteatoma, IL-2: interleukin-2, IL-9: interleukin-9.

Table 1 shows the measurements of the expression of IL-2 and IL-9, as well as CK-13 in the 16 patients (Table 1).

Table 2 shows the difference in cytokine expression in the problem tissue as statistically significant between both groups. For Group A, the average IL levels were as follows (the median is reported in parentheses): IL-2; $36.6 \pm 22.94(28)$ and IL-9, $7.4 \pm 12.13$ (7). In Group B, the average IL levels were IL-2; $73.5 \pm 10.87$ (274) and IL-9, $36.4 \pm 12.13$ (34) (Table 2).

Figures 3 and 4 show that the expression of IL-2 and IL-9 is greater in Group B than in Group A, with greater expression in the problem tissue than in the control tissue. CK-13 is also statistically different in both problem groups (Fig. 5). IL are elevated in patients with cholesteatoma.

\section{Discussion}

Cholesteatoma is one of the multiple complications of CSOM, which is characterized by bone destruction 


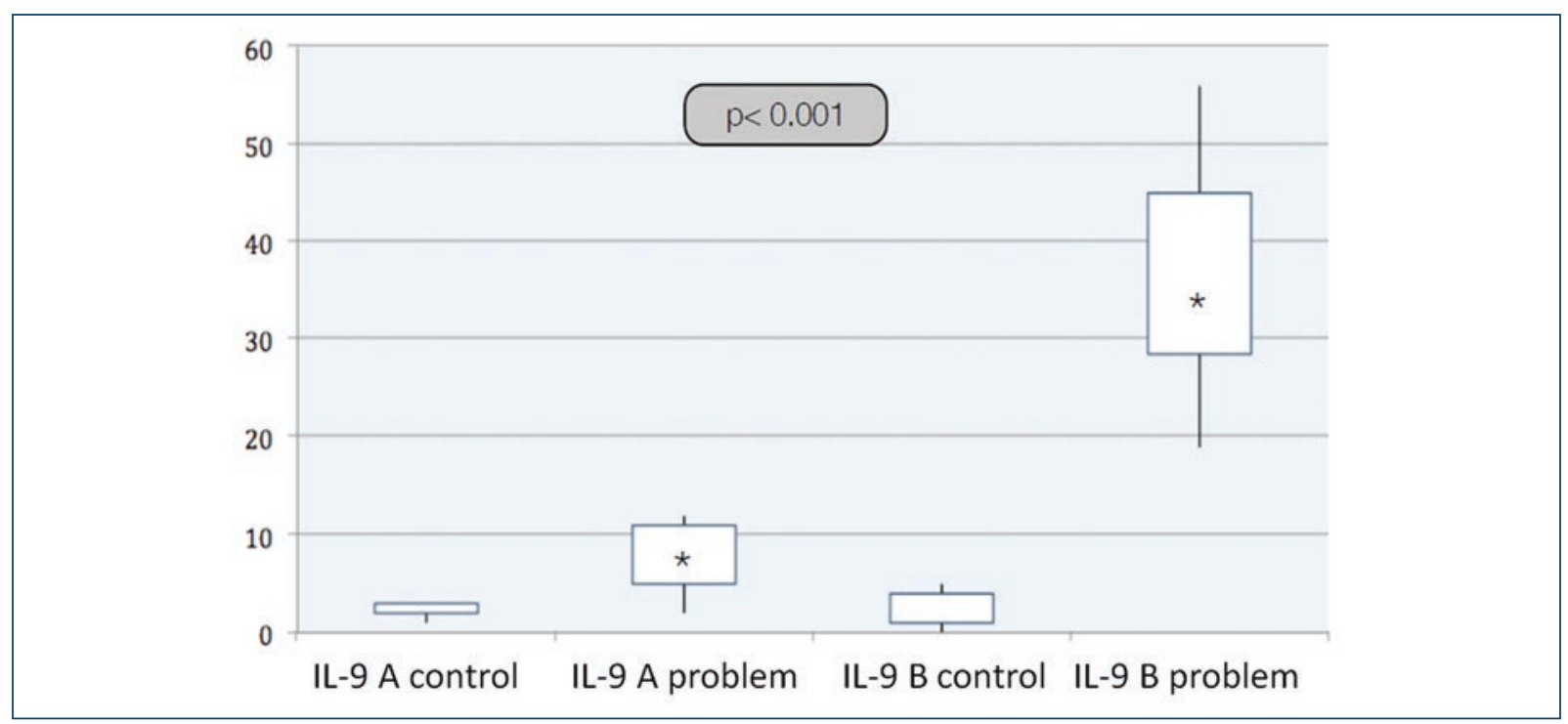

Figure 4. Interleukin-9 comparison, both groups.

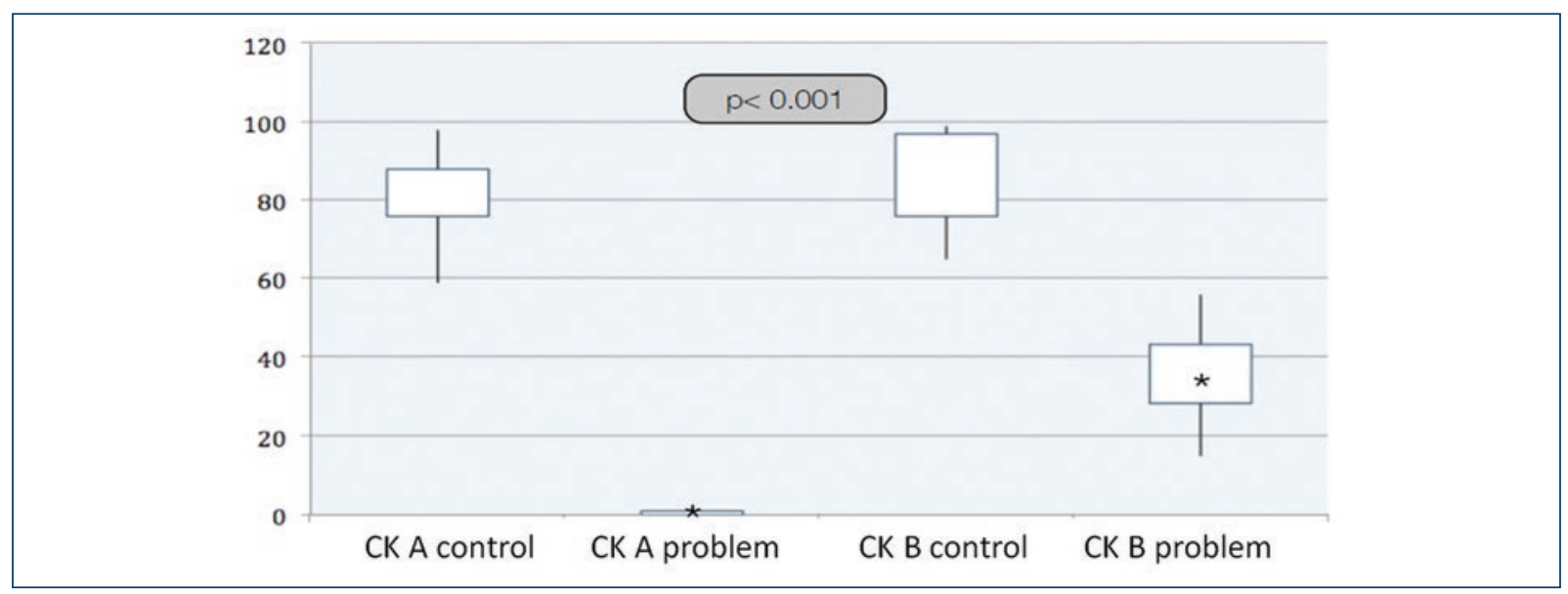

Figure 5. CK-13 comparison, both groups.

and stratified squamous epithelium replacement. The pathogenesis and pathophysiology of cholesteatoma have not been fully clarified yet; however, inflammatory mediators play a central role in its development.

During the period of this study, no IL-2 or IL-9-focused studies were found in literature. IL-2 is a growth factor as well as being a factor of survival and differentiation originated in T-lymphocytes and for T-lymphocytes. It promotes the activation of NK cells and the proliferation of B-lymphocytes. IL-9 also originates in T-lymphocytes, promotes the expression of high-affinity lgE receptors, and promotes mucus hypersecretion.

The present study showed that IL-2 and IL-9 are elevated in cholesteatomatous tissue. IL-2 can explain the persistence of T-lymphocytes and their differentiation in tissue. The presence of IL-9 can explain otorrhea, as it promotes mucosal hypersecretion in conjunction with IL-5.

This study shows that the cholesteatomatous tissue has a high presence of cytokines. Further studies are necessary to determine the degree of association between IL-2 and IL-9 levels and the degree of T-lymphocyte proliferation and otorrhea that is observed in CSOM with cholesteatoma.

\section{Conclusions}

Advances in the study of molecular biology techniques and other techniques, such as digital image 
analysis, allow for the study of several pathologies, including cholesteatoma.

The present study showed that the association of IL-2 and IL-9 with cholesteatoma is statistically significant, being high in cholesteatomatous tissues compared with control tissues.

The morphometric study by digital analysis is useful in the diagnosis of cholesteatoma because it allows determining the presence of cytokines and other molecules in the tissue quantitatively.

More studies are required to prove the participation of IL-2 and IL-9 in cholesteatoma, as well as the degree to which these are factors for the persistence and progression of cholesteatoma.

\section{Conflicts of interest}

The authors declare that there are no conflicts of interest.

\section{Acknowledgments}

The authors would like to thank the patients who kindly agreed to participate in this research and especially the Research Department of the General Hospital of Mexico for their invaluable support.

\section{Ethical disclosures}

Protection of human and animal subjects. The authors declare that the procedures followed were in accordance with the regulations of the relevant clinical research ethics committee and with those of the Code of Ethics of the World Medical Association (Declaration of Helsinki).

Confidentiality of data. The authors declare that they have followed the protocols of their work center on the publication of patient data.

Right to privacy and informed consent. The authors have obtained the written informed consent of the patients or subjects mentioned in the article. The corresponding author is in possession of this document.

\section{References}

1. Smith JA, Danner CJ. Complications of chronic otitis media and cholesteatoma. Otolaryngol Clin North Am. 2006;39:1237-55.

2. Maranhão AS, Andrade JS, Godofredo VR, Matos RC, Nde OP. Intratemporal complications of otitis media. Braz J Otorhinolaryngol. 2013;79:141-9.

3. Juhn SK, Jung MK, Hoffman MD, Drew BR, Preciado DA, Sausen NJ, et al. The role of inflammatory mediators in the pathogenesis of otitis media and sequelae. Clin Exp Otorhinolaryngol. 2008:1:117-38.

4. Aquino JE, Filho NA, De Aquino JN. Epidemiology of middle ear and mastoid cholesteatomas: study of 1146 cases. Braz J Otorhinolaryngol. 2011;77:341-7.

5. Spilsbury K, Miller I, Semmens JB, Lannigan FJ. Factors associated with developing cholesteatoma: a study of 45,980 children with middle ear disease. Laryngoscope. 2010;120:625-30.

6. Maniu A, Harabagiu O, Schrepler MP, Cătană A, F『nuță B, Mogoantă CA, et al. Molecular biology of cholesteatoma. Rom J Morphol Embryol. 2014;55:7-13.

7. Ricciardiello F, Cavaliere M, Mesolella M, lengo M. Notes on the microbiology of cholesteatoma: clinical findings and treatment. Acta Otorhinolaryngol Ital. 2009;29:197-202.

8. Alves AL, De Andrade Quintanilha Ribeiro F. The role of cytokines in acquired middle ear cholesteatoma: literature review. Rev Bras Otorrinolaringol. 2004;70:813-8.

9. Marenda SA, Aufdemorte TB. Localization of cytokines in cholesteatoma tissue. Otolaryngol Head Neck Surg. 1995;112:359-68.

10. Khan MM. Role of cytokines. In: immunopharmacology. $1^{\text {st }}$ ed. New York, US: Springer; 2008. p. 33-5.

11. Vercruysse JP, De Foer B, Somers Th, Casselman J, Offeciers E. Magnetic resonance imaging of cholesteatoma: an update. B-ENT. 2009;5:233-40.

12. Dhepnorrarat RC, Wood B, Rajan GP. Postoperative non-echo-planar diffusion-weighted magnetic resonance imaging changes after cholesteatoma surgery: implications for cholesteatoma screening. Otol Neurotol. 2009;30:54-8. 